Cochrane Database of Systematic Reviews

\title{
Treatment for hepatorenal syndrome in people with decompensated liver cirrhosis: a network meta-analysis (Protocol)
}

Best LMJ, Freeman S, Sutton AJ, Hawkins N, Tsochatzis E, Gurusamy KS

Best LMJ, Freeman S, Sutton AJ, Hawkins N, Tsochatzis E, Gurusamy KS.

Treatment for hepatorenal syndrome in people with decompensated liver cirrhosis: a network meta-analysis. Cochrane Database of Systematic Reviews 2018, Issue 9. Art. No.: CD013103.

DOI: 10.1002/14651858.CD013103.

www.cochranelibrary.com

Treatment for hepatorenal syndrome in people with decompensated liver cirrhosis: a network meta-analysis (Protocol) Copyright $\odot 2018$ The Cochrane Collaboration. Published by John Wiley \& Sons, Ltd. 


\section{TABLE OF CONTENTS}

HEADER . . . . . . . . . . . . . . . . . . . . . . . . . . . . . . . . . . . . . . . . 1

ABSTRACT . . . . . . . . . . . . . . . . . . . . . . . . . . . . . . . . . . . . . . . 1

BACKGROUND . . . . . . . . . . . . . . . . . . . . . . . . . . . . . . . . . . . . . . . 1

OBJECTIVES . . . . . . . . . . . . . . . . . . . . . . . . . . . . . . . . . . . . . .

METHODS . . . . . . . . . . . . . . . . . . . . . . . . . . . . . . . . . . . . . . 4

ACKNOWLEDGEMENTS . . . . . . . . . . . . . . . . . . . . . . . . . . . . . . . . . . . . . . . . .

REFERENCES . . . . . . . . . . . . . . . . . . . . . . . . . . . . . . . . . . . . . . . . 10

ADDITIONAL TABLES . . . . . . . . . . . . . . . . . . . . . . . . . . . . . . . . . . . . . . . . . . . . .

APPENDICES . . . . . . . . . . . . . . . . . . . . . . . . . . . . . . . . . . . . .

CONTRIBUTIONS OF AUTHORS . . . . . . . . . . . . . . . . . . . . . . . . . . . . . . . . . . . . . . . . . .

DECLARATIONS OF INTEREST . . . . . . . . . . . . . . . . . . . . . . . . . . . . . . . . . . 15

SOURCES OF SUPPORT . . . . . . . . . . . . . . . . . . . . . . . . . . . . . . . . . . . . . . . . . . . . . . . . 
[Intervention Protocol]

\title{
Treatment for hepatorenal syndrome in people with decompensated liver cirrhosis: a network meta-analysis
}

\author{
Lawrence MJ Best ${ }^{1}$, Suzanne Freeman ${ }^{2}$, Alex J Sutton ${ }^{3}$, Neil Hawkins ${ }^{4}$, Emmanuel Tsochatzis ${ }^{5}$, Kurinchi Selvan Gurusamy ${ }^{1}$ \\ ${ }^{1}$ Department of Surgery, Royal Free Campus, UCL Medical School, London, UK. ${ }^{2}$ Department of Health Sciences, College of Life \\ Sciences, Leicester, UK. ${ }^{3}$ Department of Health Sciences, University of Leicester, Leicester, UK. ${ }^{4}$ HEHTA, University of Glasgow, \\ Glasgow, UK. ${ }^{5}$ Sheila Sherlock Liver Centre, Royal Free Hospital and the UCL Institute of Liver and Digestive Health, London, UK
}

Contact address: Kurinchi Selvan Gurusamy, Department of Surgery, Royal Free Campus, UCL Medical School, Rowland Hill Street, London, NW32PF, UK. k.gurusamy@ucl.ac.uk.

Editorial group: Cochrane Hepato-Biliary Group.

Publication status and date: New, published in Issue 9, 2018.

Citation: Best LMJ, Freeman S, Sutton AJ, Hawkins N, Tsochatzis E, Gurusamy KS. Treatment for hepatorenal syndrome in people with decompensated liver cirrhosis: a network meta-analysis. Cochrane Database of Systematic Reviews 2018, Issue 9. Art. No.: CD013103. DOI: 10.1002/14651858.CD013103.

Copyright (C) 2018 The Cochrane Collaboration. Published by John Wiley \& Sons, Ltd.

\begin{abstract}
A B S T R A C T
This is a protocol for a Cochrane Review (Intervention). The objectives are as follows:

To compare the benefits and harms of different treatments of hepatorenal syndrome in people with decompensated liver cirrhosis.
\end{abstract}

\section{B A C K G R O U N D}

\section{Description of the condition}

\section{Liver cirrhosis}

The liver is a complex organ with multiple functions including carbohydrate metabolism, fat metabolism, protein metabolism, drug metabolism, synthetic functions, storage functions, digestive functions, excretory functions, and immunological functions (Read 1972). Liver cirrhosis is a liver disease in which the normal microcirculation, the gross vascular anatomy, and the hepatic architecture have been variably destroyed and altered with fibrous septa surrounding regenerated or regenerating parenchymal nodules (Tsochatzis 2014; NCBI 2018). The major causes of liver cirrhosis include excessive alcohol consumption, viral hepatitis, non-alcohol related fatty liver disease, autoimmune liver disease, and metabolic liver disease (Williams 2014; Ratib 2015; Setiawan 2016). The global prevalence of liver cirrhosis is difficult to estimate as most estimates correspond to chronic liver disease (which includes liver fibrosis and liver cirrhosis). In studies from the USA, the prevalence of chronic liver disease varies between $0.3 \%$ to $2.1 \%$ (Scaglione 2015; Setiawan 2016); in the UK, the prevalence was $0.1 \%$ in one study (Fleming 2008). In 2010, liver cirrhosis was responsible for an estimated $2 \%$ of all global deaths, equivalent to 1 million deaths (Mokdad 2014). There is an increasing trend of cirrhosis-related deaths in some countries like the UK, while there is a decreasing trend in other countries like France (Mokdad 2014; Williams 2014). The major cause of complications and deaths in people with liver cirrhosis is due to the development of clinically significant portal hypertension (hepatic venous pressure gradient at least $10 \mathrm{mmHg}$ ) (de Franchis 2015). Some of the clinical features of decompensation include jaundice, coagulopathy, ascites,

Treatment for hepatorenal syndrome in people with decompensated liver cirrhosis: a network meta-analysis (Protocol)

Copyright ( 2018 The Cochrane Collaboration. Published by John Wiley \& Sons, Ltd. 
variceal bleeding, hepatic encephalopathy, and renal failure (de Franchis 2015; McPherson 2016; EASL 2018). Decompensated cirrhosis is the most common indication for liver transplantation (Merion 2010; Adam 2012).

\section{Hepatorenal syndrome}

Hepatorenal syndrome is renal failure in people with cirrhosis in the absence of other causes of renal failure such as nephrotoxic drugs and underlying renal pathology (Angeli 2015). The current criteria for the diagnosis of hepatorenal syndrome are provided in Table 1 (Angeli 2015). Hepatorenal syndrome can be classified into type I and type II hepatorenal syndrome. Type I hepatorenal syndrome has a rapidly progressive reduction in renal function, while type II hepatorenal syndrome does not follow a rapidly progressive course (Arroyo 1996). Type I hepatorenal syndrome is associated with acute kidney injury, while type II hepatorenal syndrome is associated with chronic kidney disease (Wong 2011). However, the most recent diagnostic criteria of hepatorenal syndrome include acute kidney injury (Angeli 2015), that is most individuals classified as having hepatorenal syndrome per the current definition will fall under the type I hepatorenal syndrome of past definitions. Approximately $10 \%$ of patients hospitalised for other complications of cirrhosis develop hepatorenal syndrome (Dong 2016). Approximately $30 \%$ to $60 \%$ of people hospitalised for hepatorenal syndrome die within a year (Israelsen 2017). The annual direct medical costs of treatment of hepatorenal syndrome range between approximately USD 3 billion (3,000 million) and USD 3.8 billion (3,800 million) (Rice 2017).

\section{Pathophysiology of hepatorenal syndrome}

Portal hypertension causes arterial vasodilatation of the splanchnic circulation (dilation of the blood vessels supplying the digestive organs in the abdomen such as liver, pancreas, and intestines) (Gines 2009). This decreases the intravascular volume. In the early stages of portal hypertension, the body maintains arterial blood pressure by increasing the cardiac output; however, in later stages of portal hypertension, the increase in cardiac output is not sufficient to ensure sufficient blood supply to vital organs, and the body maintains arterial blood pressure by the activation of vasoconstrictor mechanisms (Gines 2009). These vasoconstrictor mechanisms include the renin-angiotensin system, the sympathetic nervous system, and non-osmotic hypersecretion of antidiuretic hormone (Gines 2009), and lead to decreased blood flow to the kidneys by renal arterial vasoconstriction, and eventually to renal failure (Gines 2009).

\section{Description of the intervention}

Development of hepatorenal syndrome is considered one of the manifestations of end-stage liver disease, which is one of the in- dications for liver transplantation (EASL 2016). Liver transplantation is considered the definitive treatment for hepatorenal syndrome in people who can undergo liver transplantation (Gines 2009; Acevedo 2017; EASL 2018). Supportive measures like treatment of the precipitating cause of renal failure, such as infections or gastrointestinal bleeding and fluid overload, should be provided to people during waiting time for liver transplantation and to people who cannot undergo liver transplantation due to contraindications (e.g. metastatic liver disease) (Gines 2009; EASL 2016). In addition, treatment of hepatorenal syndrome in the form of systemic vasoconstrictor drugs such as vasopressin analogues or noradrenaline, as well as renal vasodilator drugs such as dopamine, albumin, transjugular intrahepatic portosystemic shunt (TIPS), liver support with molecular adsorbent recirculating system (MARS), and renal replacement therapy in the form of haemodialysis or haemofiltration have been used while waiting for liver transplantation or in people in whom transplantation cannot be performed (Gines 2009; Hinojosa-Azaola 2014; Acevedo 2017; Allegretti 2017; EASL 2018).

\section{How the intervention might work}

Systemic vasoconstrictor drugs decrease the systemic vasodilation, which is one of the mechanisms of developing hepatorenal syndrome. Renal vasodilator drugs decrease the renal vasoconstriction, which is one of the mechanisms of developing hepatorenal syndrome. Decreased intravascular volume is one of the mechanisms of developing hepatorenal syndrome; albumin may increase the intravascular oncotic pressure and prevent third-space loss, resulting in maintenance of the intravascular volume (Caironi 2009). Transjugular intrahepatic portosystemic shunt results in a reduction of portal hypertension, which is one of the mechanisms of developing hepatorenal syndrome. Liver support with MARS and renal replacement therapy can be considered as bridging measures to prevent further deterioration of patients until the time of liver transplantation, or recovery from the precipitating factors (e.g. infections or gastrointestinal bleeding).

\section{Why it is important to do this review}

It is important to provide optimal treatment to people with hepatorenal syndrome to improve their clinical outcomes while waiting for liver transplantation or potentially prevent the need for transplantation, or both. This is particularly important given the shortage of donor organs. Several different treatments are available; however, their relative efficacy and optimal combination are not known. There have been two Cochrane Reviews on hepatorenal syndrome treatment (Allegretti 2017; Israelsen 2017); however, there have been no previous network meta-analyses on the topic. Network meta-analysis allows for a combination of direct and indirect evidence and the ranking of different interventions 
for different outcomes (Salanti 2011; Salanti 2012). With this systematic review and network meta-analysis, we aim to provide the best level of evidence for the benefits and harms of different treatments for hepatorenal syndrome in people with decompensated liver cirrhosis. If it is not possible to perform this review with network meta-analysis methods, we will instead use standard Cochrane methods to perform head-to-head comparison metaanalysis whenever possible. We will also present results from direct comparisons whenever possible, even if we perform the network meta-analysis.

\section{O B J E C T I VES}

To compare the benefits and harms of different treatments of hepatorenal syndrome in people with decompensated liver cirrhosis.

\section{METHODS}

\section{Criteria for considering studies for this review}

\section{Types of studies}

We will only consider randomised clinical trials for this network meta-analysis irrespective of language, publication status, or date of publication. We will exclude studies of other designs due to the risk of bias in such studies. Inclusion of indirect observational evidence could weaken our network meta-analysis, but this could also be viewed as a strength for assessing rare adverse events. It is well established that exclusion of non-randomised studies increases the focus on potential benefits and reduces the focus on the risks of serious adverse events and those of any adverse events. However, due to the exponentially increased amount of work required for non-randomised studies, we will register and perform a new systematic review and meta-analysis of non-randomised studies for adverse events if there is uncertainty in the balance of benefits and harms of effective treatment(s).

\section{Types of participants}

We will include randomised clinical trials with adult trial participants undergoing treatment for hepatorenal syndrome with decompensated liver cirrhosis. We will exclude randomised clinical trials in which participants had previously undergone liver transplantation.

\section{Types of interventions}

We will include any of the following interventions for comparison with one another, either alone or in combination.

- Noradrenaline (systemic vasoconstrictor)

- Terlipressin (systemic vasoconstrictor)

- Midodrine (systemic vasoconstrictor)

- Dopamine (renal vasodilator)

- Prostaglandins (renal vasodilator)

- Albumin (maintain intravascular volume)

- TIPS procedure (decrease portal hypertension)

- Other forms of portosystemic shunt (decrease portal hypertension)

- Haemodialysis (renal replacement therapy)

- Haemofiltration (renal replacement therapy)

- MARS (liver support)

- No active intervention (no intervention or placebo)

The above list is not exhaustive. If we identify treatments of which we were unaware, we will consider eligibility of the treatments for inclusion in the network if they are used primarily for treatment of hepatorenal syndrome. We will report the findings for these interventions in the 'Results' and 'Discussion' sections of the review. We will evaluate the plausibility of transitivity assumption by looking at the inclusion and exclusion criteria in the studies. Transitivity assumption is the assumption that participants included in the different trials with different treatments for hepatorenal syndrome can be considered to be a part of a multi-arm randomised clinical trial and could potentially have been randomised to any of the interventions (Salanti 2012). In other words, any participant that meets the inclusion criteria is, in principle, equally likely to be randomised to any of the above eligible interventions. This necessitates that information on potential effect-modifiers such as type of hepatorenal syndrome (type I or type II) and the co-interventions (use of prophylactic antibiotics) are the same across trials.

\section{Types of outcome measures}

\section{Primary outcomes}

- All-cause mortality at maximal follow-up (time to death).

- Health-related quality of life using a validated scale such as the EQ-5D or 36-Item Short Form Health Survey (SF-36) at maximal follow-up (EuroQol 2018; Optum 2018).

- Serious adverse events (during or within six months after cessation of intervention). We define a serious adverse event as any event that would increase mortality; is life-threatening; requires hospitalisation; results in persistent or significant disability; is a congenital anomaly/birth defect; or any important medical event that might jeopardise the person or require intervention to prevent it (ICH-GCP 1997). However, we will use the definitions used by the study authors for serious adverse events. 
- Proportion of people with one or more serious adverse events.

- Number of serious adverse events per participant.

\section{Secondary outcomes}

- Any adverse events (during or within six months after cessation of intervention). We define an adverse event as any untoward medical occurrence not necessarily having a causal relationship with the intervention but resulting in a dose reduction or discontinuation of intervention (any time after commencement of intervention) (ICH-GCP 1997). However, we will use the definition used by the study authors for adverse events.

- Proportion of people with one or more adverse events.

- Number of any adverse events per participant.

- Time to liver transplantation (maximal follow-up).

- Time to recovery from hepatorenal syndrome (maximal follow-up).

- Symptomatic recovery.

- Recovery as per definitions used for hepatorenal syndrome.

- Time to other features of decompensation (maximal followup).

\section{Exploratory outcomes}

- Length of hospital stay (all hospital admissions until maximal follow-up).

- Number of days of lost work (in people who work) (maximal follow-up).

- Treatment costs (including the cost of the treatment and any resulting complications).

We have chosen outcomes based on their importance to patients in a survey related to research priorities for people with liver diseases (Gurusamy 2018); on feedback of the patient and public representative of this project; and on an online survey about the outcomes promoted through Cochrane Consumer Network.

\section{Search methods for identification of studies}

\section{Electronic searches}

We will search the Cochrane Central Register of Controlled Trials (CENTRAL) in the Cochrane Library, MEDLINE Ovid, Embase Ovid, and Science Citation Index Expanded (Web of Science) from inception to date of search for randomised clinical trials comparing two or more of the above interventions, applying no language restrictions (Royle 2003). We will search for all possible comparisons formed by the interventions of interest. To identify further ongoing or completed trials, we will also search the US National Institutes of Health Ongoing Trials Register ClinicalTrials.gov (clinicaltrials.gov) and the World Health Organization International Clinical Trials Registry Platform ( WHO ICTRP) ( apps.who.int/trialsearch/), which searches various trial registers, including ISRCTN and ClinicalTrials.gov. We will also search the European Medicines Agency (EMA) (www.ema.europa.eu/ema/) and US Food and Drug Administration ( FDA) ( www.fda.gov) registries for randomised clinical trials. The provisional search strategies are provided in Appendix 1.

\section{Searching other resources}

We will search the references of the identified trials and the existing Cochrane Reviews on hepatorenal syndrome to identify additional trials for inclusion.

\section{Data collection and analysis}

\section{Selection of studies}

Two review authors (KG and LB) will independently screen the titles and abstracts of studies identified by the search for potential inclusion in the review. We will seek full-text articles for any references identified by at least one of the review authors as potentially relevant and assess trials for inclusion based on the full-text articles. We will provide a list of excluded references and the reasons for their exclusion in the 'Characteristics of excluded studies' table. We will also list any ongoing trials identified primarily through the search of the clinical trial registers for further follow-up. Any discrepancies will be resolved through discussion.

\section{Data extraction and management}

Two review authors (KG and LB) will independently extract the data below in a pre-piloted Microsoft Excel-based data extraction form (after translation of non-English articles).

- Outcome data (for each outcome and for each intervention group whenever applicable):

$\circ$ number of participants randomised;

- number of participants included for the analysis;

- number of participants with events for binary outcomes, mean and standard deviation for continuous outcomes, number of events and the mean follow-up period for count outcomes, and number of participants with events and the mean follow-up period for time-to-event outcomes;

$\circ$ natural logarithm of hazard ratio and its standard error if this was reported rather than the number of participants with events and the mean follow-up period for time-to-event outcomes;

$\circ$ definition of outcomes or scale used if appropriate. 
- Data on potential effect modifiers:

- participant characteristics such as age, sex, definition and type of hepatorenal syndrome (type I or type II), the aetiology for cirrhosis, and the interval between diagnosis of hepatorenal syndrome and treatment;

$\circ$ details of the intervention and control (including dose, frequency, and duration);

○ length of follow-up;

○ information related to 'Risk of bias' assessment (see

Assessment of risk of bias in included studies).

- Other data:

$\circ$ year and language of publication;

o country in which the participants were recruited;

- year(s) in which the trial was conducted;

$\circ$ inclusion and exclusion criteria.

We will collect outcomes at maximum follow-up but also at shortterm (up to three months) and medium-term (more than three months to five years) if this is available.

We will contact the trial authors in the case of unclear or missing information. If there is any doubt as to whether trials shared the same participants, completely or partially (by identifying common authors and centres), we will attempt to contact the trial authors to clarify whether the trial report was duplicated. Any differences in opinion will be resolved through discussion.

\section{Assessment of risk of bias in included studies}

We will follow the guidance in the Cochrane Handbook for Systematic Reviews of Interventions and that described in the Cochrane Hepato-Biliary Group Module to assess the risk of bias in included trials (Higgins 2011; Gluud 2018). Specifically, we will assess sources of bias as defined below (Schulz 1995; Moher 1998; Kjaergard 2001; Wood 2008; Savović 2012a; Savović 2012b; Lundh 2017; Savović 2018).

\section{Allocation sequence generation}

- Low risk of bias: the study authors performed sequence generation using computer random number generation or a random number table. Drawing lots, tossing a coin, shuffling cards, and throwing dice are adequate if performed by an independent person not otherwise involved in the study. In general, we will classify the risk of bias is low if the method used for allocation concealment suggested that it was extremely likely that the sequence was generated randomly (e.g. the use of an interactive voice response system).

- Unclear risk of bias: the study authors did not specify the method of sequence generation.

- High risk of bias: the sequence generation method was not random.

\section{Allocation concealment}

- Low risk of bias: the participant allocations could not have been foreseen in advance of, or during, enrolment. A central and independent randomisation unit controlled allocation. The investigators were unaware of the allocation sequence (e.g. if the allocation sequence was hidden in sequentially numbered, opaque, and sealed envelopes).

- Unclear risk of bias: the study authors did not describe the method used to conceal the allocation so that the intervention allocations may have been foreseen before, or during, enrolment.

- High risk of bias: it is likely that the investigators who assigned the participants knew the allocation sequence. We will exclude such quasi-randomised studies.

\section{Blinding of participants and personnel}

- Low risk of bias: any of the following: blinding of participants and key study personnel ensured, and it was unlikely that the blinding could have been broken; or rarely no blinding or incomplete blinding, but the review authors judged that the outcome was not likely to be influenced by lack of blinding.

- Unclear risk of bias: any of the following: insufficient information to permit judgement of 'low risk' or 'high risk'; or the trial did not address this outcome.

- High risk of bias: any of the following: no blinding or incomplete blinding, and the outcome was likely to be influenced by lack of blinding; or blinding of key study participants and personnel attempted, but likely that the blinding could have been broken, and the outcome was likely to be influenced by lack of blinding.

\section{Blinded outcome assessment}

- Low risk of bias: any of the following: blinding of outcome assessment ensured, and unlikely that the blinding could have been broken; or rarely no blinding of outcome assessment, but the review authors judged that the outcome measurement was not likely to be influenced by lack of blinding.

- Unclear risk of bias: any of the following: insufficient information to permit judgement of 'low risk' or 'high risk'; or the trial did not address this outcome.

- High risk of bias: any of the following: no blinding of outcome assessment, and the outcome measurement was likely to be influenced by lack of blinding; or blinding of outcome assessment, but likely that the blinding could have been broken, and the outcome measurement was likely to be influenced by lack of blinding.

\section{Incomplete outcome data}

- Low risk of bias: missing data were unlikely to make treatment effects depart from plausible values. The study used 
sufficient methods, such as multiple imputation, to handle missing data.

- Unclear risk of bias: there was insufficient information to assess whether missing data in combination with the method used to handle missing data were likely to induce bias on the results.

- High risk of bias: the results were likely to be biased due to missing data.

\section{Selective outcome reporting}

- Low risk of bias: the trial reported the following predefined outcomes: at least one of the outcomes related to the main reason for treatment of people with hepatorenal syndrome, namely, mortality or resolution of hepatorenal syndrome along with adverse events. If the original trial protocol was available, the outcomes should have been those called for in that protocol. If the trial protocol was obtained from a trial registry (e.g. ClinicalTrials.gov), the outcomes sought should have been those enumerated in the original protocol if the trial protocol was registered before or at the time that the trial was begun. If the trial protocol was registered after the trial was begun, those outcomes will not be considered to be reliable.

- Unclear risk of bias: not all predefined, or clinically relevant and reasonably expected, outcomes were reported fully, or it was unclear whether data on these outcomes were recorded or not.

- High risk of bias: one or more predefined or clinically relevant and reasonably expected outcomes were not reported, despite the fact that data on these outcomes should have been available and even recorded.

\section{For-profit bias}

- Low risk of bias: the trial appeared to be free of industry sponsorship or other type of for-profit support that could manipulate the trial design, conductance, or results of the trial (industry-sponsored trials overestimate the efficacy by about 25\%) (Lundh 2017).

- Unclear risk of bias: the trial may or may not have been free of for-profit bias, as no information on clinical trial support or sponsorship was provided.

- High risk of bias: the trial was sponsored by industry or received another type of for-profit support.

\section{Other bias}

- Low risk of bias: the trial appeared to be free of other components that could put it at risk of bias (e.g. inappropriate control or dose or administration of control, baseline differences, early stopping).

- Unclear risk of bias: the trial may or may not have been free of other components that could put it at risk of bias.
- High risk of bias: there were other factors in the trial that could put it at risk of bias (e.g. baseline differences, early stopping).

We will consider a trial to be at low risk of bias if we assess the trial to be at low risk of bias across all listed 'Risk of bias' domains. Otherwise, we will consider trials to be at high risk of bias. At the outcome level, we will classify an outcome to be at low risk of bias if the allocation sequence generation; allocation concealment; blinding of participants, healthcare professionals, and outcome assessors; incomplete outcome data; and selective outcome reporting (at the outcome level) are at low risk of bias for objective and subjective outcomes (Savović 2018).

\section{Measures of treatment effect}

Relative treatment effects

For dichotomous variables (e.g. the proportion of participants with serious adverse events or any adverse event), we will calculate the odds ratio (OR) with 95\% credible interval (CrI) (or Bayesian confidence interval) (Severini 1993). For continuous variables (e.g. health-related quality of life reported on the same scale), we will calculate the mean difference (MD) with $95 \% \mathrm{Crl}$. We will use standardised mean difference (SMD) values with 95\% Crl for health-related quality of life if the included trials used different scales. For count outcomes (e.g. number of serious adverse events or number of any adverse event), we will calculate the rate ratio (RaR) with 95\% Crl. For time-to-event data (e.g. all-cause mortality at maximal follow-up), we will calculate hazard ratio (HR) with $95 \% \mathrm{Crl}$.

Relative ranking

We will estimate the ranking probabilities for all interventions of being at each possible rank for each intervention. We will obtain the surface under the cumulative ranking curve (SUCRA) (cumulative probability), rankogram, and relative ranking table with $\mathrm{CrI}$ for the ranking probabilities (Salanti 2011; Chaimani 2013).

\section{Unit of analysis issues}

The unit of analysis is the participant undergoing treatment for hepatorenal syndrome according to the intervention group to which the participant was randomly assigned.

\section{Cluster randomised clinical trials}

We will include cluster randomised clinical trials provided that the effect estimate adjusted for cluster correlation is available. If this is not available, we will include such trials if sufficient information to calculate the design effect is available from the trial because this will allow us to take clustering into account. We will also assess additional domains of risk of bias for cluster randomised trials according to guidance in the Cochrane Handbook for Systematic Reviews of Interventions (Higgins 2011). 


\section{Cross-over randomised clinical trials}

If we identify any cross-over randomised clinical trials, we will include the outcomes after the period of first intervention because the included treatments can have residual effects.

\section{Trials with multiple intervention groups}

We will collect data for all trial intervention groups that meet the inclusion criteria. The codes we will use for analysis will account for the correlation between the effect sizes from studies with more than two groups.

\section{Dealing with missing data}

We will perform an intention-to-treat analysis whenever possible (Newell 1992); otherwise, we will use the data available to us. This may result in the use of 'per-protocol' analyses. Since these may be biased, particularly if the data are not missing at random (e.g. treatment was withdrawn due to adverse events, or duration of treatment was shortened due to lack of response and such participants were excluded from analysis), we will conduct bestworst-case scenario analysis (good outcome in intervention group and bad outcome in control group) and worst-best-case scenario analysis (bad outcome in intervention group and good outcome in control group) as sensitivity analyses whenever possible for dichotomous outcomes.

For continuous outcomes, we will impute the standard deviation from P values according to guidance in the Cochrane Handbook for Systematic Reviews of Interventions (Higgins 2011). If the data are likely to be normally distributed, we will use the median for meta-analysis when the mean is not available. If it is not possible to calculate the standard deviation from the P value or the confidence intervals, we will impute the standard deviation using the largest standard deviation in other trials for that outcome. This form of imputation can decrease the weight of the study for calculation of mean differences and may bias the effect estimate to no effect for calculation of standardised mean differences (Higgins 2011).

\section{Assessment of heterogeneity}

We will assess clinical and methodological heterogeneity by carefully examining the characteristics and design of included trials. We will assess the presence of clinical heterogeneity by comparing effect estimates (see Subgroup analysis and investigation of heterogeneity) in trial reports of different drug dosages, different types of hepatorenal syndrome (type I and type II), different aetiologies for cirrhosis (e.g. alcohol-related liver disease, viral liver diseases, autoimmune liver disease), and based on the co-interventions (e.g. both groups receive prophylactic antibiotics to decrease the risk of subacute bacterial peritonitis). Different study designs and risk of bias can contribute to methodological heterogeneity. We will assess statistical heterogeneity by comparing the results of the fixed-effect model meta-analysis and the random-effects model meta-analysis, between-study standard deviation ( $\mathrm{Tau}^{2}$ and comparing this with values reported in study of the distribution of between-study heterogeneity) (Turner 2012), and by calculating I ${ }^{2}$ using Stata/SE 14.2. If we identify substantial clinical, methodological, or statistical heterogeneity, we will explore the heterogeneity and address it in subgroup analysis (see Subgroup analysis and investigation of heterogeneity).

\section{Assessment of transitivity across treatment comparisons}

We will assess the transitivity assumption by comparing the distribution of the potential effect modifiers (clinical: type of hepatorenal syndrome (type I versus type II); methodological: risk of bias, year of randomisation, duration of follow-up) across the different pairwise comparisons.

\section{Assessment of reporting biases}

For the network meta-analysis, we will perform a comparisonadjusted funnel plot. If there is no meaningful way in which to rank these studies (i.e. there was no specific change in the risk of bias in the studies, sample size, or the control group used over time), we will judge the reporting bias by the completeness of the search (Chaimani 2012).

\section{Data synthesis}

\section{Methods for indirect and mixed comparisons}

We will conduct network meta-analyses to compare multiple interventions simultaneously for each of the primary and secondary outcomes. Network meta-analysis combines direct evidence within trials and indirect evidence across trials (Mills 2012). We will obtain a network plot to ensure that the trials are connected by interventions using Stata/SE 14.2 (Chaimani 2013). We will exclude any trials that are not connected to the network from the network meta-analysis and report only the direct pairwise meta-analysis for such comparisons. We will summarise the population and methodological characteristics of the trials included in the network meta-analysis in a table based on pairwise comparisons. We will conduct a Bayesian network meta-analysis using the Markov chain Monte Carlo method in OpenBUGS 3.2.3 as per guidance from the National Institute for Health and Care Excellence (NICE) Decision Support Unit (DSU) documents (Dias 2016). We will model the treatment contrast (i.e. log odds ratio for binary outcomes, mean difference or standardised mean difference for continuous outcomes, $\log$ rate ratio for count outcomes, and $\log$ hazard ratio for time-to-event outcomes) for any two interventions ('functional parameters') as a function of comparisons between each individual intervention and the reference group ('basic parameters') using appropriate likelihood functions and links ( $\mathrm{Lu}$ 
2006). We will use binomial likelihood and logit link for binary outcomes, Poisson likelihood and log link for count outcomes, binomial likelihood and complementary log-log link for time-toevent outcomes (a semiparametric model which excludes censored individuals from the denominator of 'at risk' individuals at the point when they are censored), and normal likelihood and identity link for continuous outcomes. We will use the 'no active intervention' as the reference group. We will perform a fixed-effect model and random-effects model for the network meta-analysis. We will report both models for comparison with the reference group in a forest plot. For each pairwise comparison in a table, we will report the fixed-effect model if the two models report similar results; otherwise, we will report the more conservative model.

We will use a hierarchical Bayesian model using three different initial values, employing codes provided by NICE DSU (Dias 2016). We will use a normal distribution with large variance $(10,000)$ for treatment effect priors (vague or flat priors). For the random-effects model, we will use a prior distributed uniformly (limits: 0 to 5) for between-trial standard deviation but will assume same between-trial standard deviation across treatment comparisons (Dias 2016). We will use a 'burn-in' of 10,000 simulations, check for convergence (of effect estimates and between-study heterogeneity) visually (i.e. whether the values in different chains mix very well by visualisation), and run the models for another 10,000 simulations to obtain effect estimates. If we do not obtain convergence, we will increase the number of simulations for the 'burn-in'. If we still do not obtain convergence, we will use alternate initial values and priors employing methods suggested by van Valkenhoef 2012. We will estimate the probability that each intervention ranks at one of the possible positions using the NICE DSU codes (Dias 2016).

\section{Assessment of inconsistency}

We will assess inconsistency (statistical evidence of the violation of transitivity assumption) by fitting both an inconsistency model and a consistency model. We will use inconsistency models employed in the NICE DSU manual, as we will use a common between-study standard deviation (Dias 2014). In addition, we will use design-by-treatment full interaction model and inconsistency factor (IF) plots to assess inconsistency (Higgins 2012; Chaimani 2013). We will use Stata/SE 14.2 to create IF plots. In the presence of inconsistency, we will assess whether the inconsistency was due to clinical or methodological heterogeneity by performing separate analyses for each of the different subgroups mentioned in the Subgroup analysis and investigation of heterogeneity section.

If there is evidence of inconsistency, we will identify areas in the network where substantial inconsistency might be present in terms of clinical and methodological diversities between trials and, when appropriate, limit network meta-analysis to a more compatible subset of trials.

\section{Direct comparison}

We will perform the direct comparisons using the same codes and the same technical details.

\section{Calculation of required information size and Trial Sequential Analysis}

For calculation of the required information size, see Appendix 2. We will perform Trial Sequential Analysis for direct comparisons to control the risk of random errors when at least two trials are included for the comparison of other interventions versus no active intervention ('control') for the outcomes all-cause mortality at maximal follow-up and health-related quality of life, the two outcomes that determine whether the intervention should be given (Wetterslev 2008; Thorlund 2011; TSA 2011; Wetterslev 2017). For all-cause mortality at maximal follow-up, we will use an alpha error as per guidance of Jakobsen 2014 (i.e. 0.033), power of $90 \%$ (beta error of 10\%) (Castellini 2017), a relative risk reduction of $20 \%$, the median control group proportion observed in the trials, and the heterogeneity observed in the meta-analysis using Stata/SE 14.2, employing methods suggested by Miladinovic 2013. For health-related quality of life, a continuous outcome, we will use an alpha error as per guidance of Jakobsen 2014 (i.e. 0.033), power of 90\% (beta error of 10\%) (Castellini 2017), a standardised mean difference of 0.2 , the median health-related quality of life in the control group in the trials, and the heterogeneity observed in the meta-analysis.

\section{Subgroup analysis and investigation of heterogeneity}

We plan to assess the differences in the effect estimates between the following subgroups and investigate heterogeneity and inconsistency using meta-regression with the help of the codes provided in NICE DSU guidance if we include a sufficient number of trials (Dias 2012a). We plan to use the following trial-level covariates for meta-regression.

- Trials at low risk of bias compared to trials at high risk of bias.

- Based on the type of hepatorenal syndrome (type I versus type II).

- Based on the aetiology for cirrhosis (e.g. alcohol-related liver disease, viral liver diseases, autoimmune liver disease).

- Based on the interval between the diagnosis of hepatorenal syndrome and the start of treatment.

- Based on the co-interventions (e.g. both groups received prophylactic antibiotics to decrease the risk of subacute bacterial peritonitis).

- Based on the period of follow-up (short-term: up to three months; medium term: more than three months to five years; long-term: more than five years).

- Based on the definition used by authors for serious adverse events and any adverse events (ICH-GCP 1997 versus other definitions). 
We will calculate a single common interaction term when applicable (Dias 2012a). If the $95 \%$ Crls of the interaction term does not overlap zero, we will consider this statistically significant heterogeneity or inconsistency (depending upon the factor being used as covariate).

\section{Sensitivity analysis}

If a trial reports only per-protocol analysis results, we plan to reanalyse the results using the best-worst-case scenario and worstbest-case scenario analyses as sensitivity analyses whenever possible. We will also perform a sensitivity analysis excluding the trials in which mean or standard deviation, or both were imputed and use the median standard deviation in the trials to impute missing standard deviations.

\section{Presentation of results}

We will follow the PRISMA for Network Meta-Analyses while reporting (Hutton 2015). We will present the effect estimates with 95\% CrI for each pairwise comparison calculated from the direct comparisons and network meta-analysis. We will also present the cumulative probability of the treatment ranks (i.e. the probability that the intervention is within the top two, the probability that the intervention is within the top three, etc.) in graphs (SUCRA) (Salanti 2011). We will plot the probability that each intervention was best, second best, third best, etc. for each of the different outcomes (rankograms), which are generally considered more informative (Salanti 2011; Dias 2012b). We will also provide the $\mathrm{CrI}$ of the probabilities in the ranking probability tables. We will upload all the raw data and the codes used for analysis in the European Organization for Nuclear Research open source database (Zenodo) and provide a link within the review.

\section{Grading of evidence}

We will present 'Summary of findings' tables for all the primary and secondary outcomes (see Primary outcomes; Secondary outcomes). We will follow the approach suggested by Puhan and colleagues (Puhan 2014). First, we will calculate the direct and indirect effect estimates and 95\% CrI using the node-splitting approach (Dias 2010), that is calculating the direct estimate for each comparison by including only trials in which there was direct comparison of interventions and the indirect estimate for each comparison by excluding the trials in which there was direct comparison of interventions. Next, we will rate the quality of direct and indirect effect estimates using GRADE methodology, which takes into account the risk of bias, inconsistency, directness of evidence, imprecision, and publication bias (Guyatt 2011). We will then present the estimates of the network meta-analysis and rate the quality of network meta-analysis effect estimates as the best quality of evidence between the direct and indirect estimates (Puhan
2014). In addition, we will present information on the absolute measures (i.e. proportion of people with the outcome in each intervention group based on the direct estimates, indirect estimates, and network meta-analysis estimates). We will also present information on the number of trials and participants as per the standard 'Summary of findings' table.

\section{Recommendations for future research}

We will also provide recommendations for future research in the population, intervention, control, outcomes, period of follow-up, and study design based on the uncertainties that we identify from the existing research.

\section{ACKNOWLEDGEMENTS}

We acknowledge the help and support of the Cochrane HepatoBiliary Group. We would also like to thank the people listed below who provided comments to improve the protocol.

Peer reviewers: Rosa Martin-Mateos, Spain; Carlos Benitez, Brazil. Contact Editor: Christian Gluud, Denmark.

Sign-off Editor: Christian Gluud, Denmark.

Cochrane Review Group funding acknowledgement: the Danish State is the largest single funder of the Cochrane Hepato-Biliary Group through its investment in the Copenhagen Trial Unit, Centre for Clinical Intervention Research, Rigshospitalet, Copenhagen University Hospital, Denmark.

This project was funded by the National Institute for Health Research Systematic Reviews Programme (project number 16/114/ 17) and was supported by the Complex Reviews Support Unit, also funded by the National Institute for Health Research (project number 14/178/29).

\section{Department of Health disclaimer}

The views and opinions expressed herein are those of the authors and do not necessarily reflect those of the National Institute for Health Research, National Health Service (NHS), or the Department of Health.

\section{Danish State and the Copenhagen Trial Unit disclaimer}

The views and opinions expressed in this review are those of the authors and do not necessarily reflect those of the Danish State or the Copenhagen Trial Unit. 


\section{REFERE N C E S}

\section{Additional references}

\section{Acevedo 2017}

Acevedo JG, Cramp ME. Hepatorenal syndrome: update on diagnosis and therapy. World Journal of Hepatology 2017; 9(6):293-9.

\section{Adam 2012}

Adam R, Karam V, Delvart V, O'Grady J, Mirza D, Klempnauer J, et al. Evolution of indications and results of liver transplantation in Europe. A report from the European Liver Transplant Registry (ELTR). Journal of Hepatology 2012;57(3):675-88.

\section{Allegretti 2017}

Allegretti A, Israelsen M, Krag A, Jovani M, Goldin A, Schulman A, et al. Terlipressin versus placebo or no intervention for people with cirrhosis and hepatorenal syndrome. Cochrane Database of Systematic Reviews 2017, Issue 6. DOI: 10.1002/14651858.CD005162.pub4

\section{Angeli 2015}

Angeli P, Gines P, Wong F, Bernardi M, Boyer TD, Gerbes A, et al. Diagnosis and management of acute kidney injury in patients with cirrhosis: revised consensus recommendations of the International Club of Ascites. Journal of Hepatology 2015;62(4):968-74.

\section{Arroyo 1996}

Arroyo V, Gines P, Gerbes AL, Dudley FJ, Gentilini P, Laffi $G$, et al. Definition and diagnostic criteria of refractory ascites and hepatorenal syndrome in cirrhosis. International Ascites Club. Hepatology (Baltimore, Md.) 1996;23(1): 164-76.

\section{Caironi 2009}

Caironi P, Gattinoni L. The clinical use of albumin: the point of view of a specialist in intensive care. Trasfusione del Sangue [Blood Transfusion] 2009;7(4):259-67.

\section{Castellini 2017}

Castellini G, Nielsen EE, Gluud C. Comment on: "Cell therapy for heart disease: trial sequential analyses of two Cochrane reviews". Clinical Pharmacology and Therapeutics 2017; Vol. 102, issue 1:21-4.

\section{Chaimani 2012}

Chaimani A, Salanti G. Using network meta-analysis to evaluate the existence of small-study effects in a network of interventions. Research Synthesis Methods 2012;3(2): 161-76.

\section{Chaimani 2013}

Chaimani A, Higgins JP, Mavridis D, Spyridonos P, Salanti G. Graphical tools for network meta-analysis in STATA. PLoS ONE 2013;8(10):e76654.

\section{de Franchis 2015}

de Franchis R, Baveno VIF. Expanding consensus in portal hypertension: report of the Baveno VI consensus workshop: stratifying risk and individualizing care for portal hypertension. Journal of Hepatology 2015;63(3):743-52.
Del Re 2013

Del Re AC, Spielmans GI, Flückiger C, Wampold BE. Efficacy of new generation antidepressants: differences seem illusory. PLoS ONE 2013;8(6):e63509.

\section{Dias 2010}

Dias S, Welton NJ, Caldwell DM, Ades AE. Checking consistency in mixed treatment comparison meta-analysis. Statistics in Medicine 2010;29(7-8):932-44.

\section{Dias 2012a}

Dias S, Sutton AJ, Welton NJ, Ades AE. NICE DSU technical support document 3: heterogeneity: subgroups, meta-regression, bias and bias-adjustment, September 2011 (last updated April 2012). nicedsu.org.uk/wpcontent/uploads/2016/03/TSD3-Heterogeneity.finalreport.08.05.12.pdf (accessed 17 July 2018).

\section{Dias 2012b}

Dias S, Welton NJ, Sutton AJ, Ades AE. NICE DSU technical support document 1: introduction to evidence synthesis for decision making, April 2011 (last updated April 2012). scharr.dept.shef.ac.uk/nicedsu/wp-content/uploads/ sites/7/2016/03/TSD1-Introduction.final_.08.05.12.pdf (accessed 17 July 2018).

\section{Dias 2014}

Dias S, Welton NJ, Sutton AJ, Caldwell DM, Lu G, Ades AE. NICE DSU technical support document 4: inconsistency in networks of evidence based on randomised controlled trials, May 2011 (last updated April 2014). nicedsu.org.uk/wp-content/uploads/2016/03/TSD4Inconsistency.final_.15April2014.pdf (accessed 17 July 2018).

\section{Dias 2016}

Dias S, Welton NJ, Sutton AJ, Ades AE. NICE DSU technical support document 2: a generalised linear modelling framework for pairwise and network meta-analysis of randomised controlled trials, August 2011 (last updated September 2016). www.ncbi.nlm.nih.gov/pubmedhealth/ PMH0088912/pdf/PubMedHealth_PMH0088912.pdf (accessed 17 July 2018).

Dong 2016

Dong T, Aronsohn A, Gautham Reddy K, Te HS. Rifaximin decreases the incidence and severity of acute kidney injury and hepatorenal syndrome in cirrhosis. Digestive Diseases and Sciences 2016;61(12):3621-6.

\section{EASL 2016}

European Association for the Study of the Liver. EASL Clinical Practice Guidelines: liver transplantation. Journal of Hepatology 2016;64(2):433-85.

\section{EASL 2018}

European Association for the Study of the Liver. EASL Clinical Practice Guidelines for the management of patients with decompensated cirrhosis. Journal of Hepatology 2018 Apr 10 Epub ahead of print]. DOI: 10.1016/ j.jhep.2018.03.024 


\section{EuroQol 2018}

EuroQol. EQ-5D Instruments | About EQ-5D, 2018. euroqol.org/eq-5d-instruments/ (accessed 17 July 2018).

\section{Fleming 2008}

Fleming KM, Aithal GP, Solaymani-Dodaran M, Card TR, West J. Incidence and prevalence of cirrhosis in the United Kingdom, 1992-2001: a general population-based study. Journal of Hepatology 2008;49(5):732-8.

Gines 2009

Gines P, Schrier RW. Renal failure in cirrhosis. New England Journal of Medicine 2009;361(13):1279-90.

Gluud 2018

Gluud C, Nikolova D, Klingenberg SL. Cochrane HepatoBiliary Group. About The Cochrane Collaboration 2018, Issue 3. Art. No.: LIVER.

Gurusamy 2018

Gurusamy K, Walmsley M, Davidson BR, Frier C, Fuller B, Madden A, et al. Top research priorities in liver and gallbladder disorders in the United Kingdom. Article under review.

\section{Guyatt 2011}

Guyatt G, Oxman AD, Akl EA, Kunz R, Vist G, Brozek J, et al. GRADE guidelines: 1. Introduction - GRADE evidence profiles and summary of findings tables. Journal of Clinical Epidemiology 2011;64(4):383-94.

\section{Higgins 2011}

Higgins JPT, Green S (editors). Cochrane Handbook for Systematic Reviews of Interventions Version 5.1.0 (updated March 2011). The Cochrane Collaboration, 2011. Available from handbook.cochrane.org.

\section{Higgins 2012}

Higgins JPT, Jackson D, Barrett JK, Lu G, Ades AE, White IR. Consistency and inconsistency in network metaanalysis: concepts and models for multi-arm studies. Research Synthesis Methods 2012;3(2):98-110.

Hinojosa-Azaola 2014

Hinojosa-Azaola A, Salas Nolasco OI, Gonzalez Garay AG, Chavez-Tapia NC, Solis Galicia C. Transjugular intrahepatic portosystemic shunts for hepatorenal syndrome. Cochrane Database of Systematic Reviews 2014, Issue 3. DOI: 10.1002/14651858.CD011039

Hutton 2015

Hutton B, Salanti G, Caldwell DM, Chaimani A, Schmid $\mathrm{CH}$, Cameron $\mathrm{C}$, et al. The PRISMA extension statement for reporting of systematic reviews incorporating network meta-analyses of health care interventions: checklist and explanations. Annals of Internal Medicine 2015;162(11): $777-84$.

\section{ICH-GCP 1997}

International Conference on Harmonisation Expert Working Group. International Conference on Harmonisation of Technical Requirements for Registration of Pharmaceuticals for Human Use. ICH Harmonised Tripartite Guideline. Guideline for Good Clinical Practice CFR \& ICH Guidelines. Vol. 1, Philadelphia (PA): Barnett International/PAREXEL, 1997.

\section{Israelsen 2017}

Israelsen M, Krag A, Allegretti AS, Jovani M, Goldin AH, Winter R W, et al. Terlipressin versus other vasoactive drugs for hepatorenal syndrome. Cochrane Database of Systematic Reviews 2017, Issue 9. DOI: 10.1002/ 14651858.CD011532.pub2

\section{Jakobsen 2014}

Jakobsen JC, Wetterslev J, Winkel P, Lange T, Gluud C. Thresholds for statistical and clinical significance in systematic reviews with meta-analytic methods. $B M C$ Medical Research Methodology 2014;14(1):120.

\section{Kjaergard 2001}

Kjaergard LL, Villumsen J, Gluud C. Reported methodologic quality and discrepancies between large and small randomized trials in meta-analyses. Annals of Internal Medicine 2001;135(11):982-9.

Lu 2006

Lu G, Ades AE. Assessing evidence inconsistency in mixed treatment comparisons. Journal of the American Statistical Association 2006;101(474):447-59.

Lundh 2017

Lundh A, Sismondo S, Lexchin J, Busuioc OA, Bero L. Industry sponsorship and research outcome. Cochrane Database of Systematic Reviews 2017, Issue 2. DOI: 10.1002/14651858.MR000033.pub3

\section{McPherson 2016}

McPherson S, Lucey MR, Moriarty KJ. Decompensated alcohol related liver disease: acute management. $B M J$ (Clinical Research Ed.) 2016;352:i124.

\section{Merion 2010}

Merion RM. Current status and future of liver transplantation. Seminars in Liver Disease 2010;30(4): 411-21.

\section{Miladinovic 2013}

Miladinovic J, Hozo I, Djulbegovic B. Trial sequential boundaries for cumulative meta-analyses. Stata Journal 2013;13(1):77-91.

\section{Mills 2012}

Mills EJ, Ioannidis JP, Thorlund K, Schunemann HJ, Puhan MA, Guyatt GH. How to use an article reporting a multiple treatment comparison meta-analysis. JAMA 2012; 308(12):1246-53.

\section{Moher 1998}

Moher D, Pham B, Jones A, Cook DJ, Jadad AR, Moher $\mathrm{M}$, et al. Does quality of reports of randomised trials affect estimates of intervention efficacy reported in meta-analyses? . Lancet 1998;352(9128):609-13.

\section{Mokdad 2014}

Mokdad AA, Lopez AD, Shahraz S, Lozano R, Mokdad AH, Stanaway J, et al. Liver cirrhosis mortality in 187 countries between 1980 and 2010: a systematic analysis. BMC Medicine 2014;12:145.

\section{NCBI 2018}

NCBI. Liver cirrhosis. www.ncbi.nlm.nih.gov/mesh/ 68008103 (accessed 17 July 2018). 


\section{Newell 1992}

Newell DJ. Intention-to-treat analysis: implications for quantitative and qualitative research. International Journal of Epidemiology 1992;21(5):837-41.

OpenBUGS 3.2.3 [Computer program]

Members of OpenBUGS Project Managemnt Group. OpenBUGS. Version 3.2.3. Members of OpenBUGS Project Managemnt Group, 15 March 2014.

Optum 2018

Optum. Patient-reported Outcomes | What We Do | SF Health Surveys | SF-36v2 Health Survey, 2018. campaign.optum.com/optum-outcomes/what-we-do/ health-surveys/sf-36v2-health-survey.html (accessed 14 April 2018).

\section{Puhan 2014}

Puhan MA, Schünemann HJ, Murad MH, Li T, Brignardello-Petersen R, Singh JA, et al. A GRADE Working Group approach for rating the quality of treatment effect estimates from network meta-analysis. BMJ (Clinical Research Ed.) 2014;349:g5630.

\section{Ratib 2015}

Ratib S, Fleming KM, Crooks CJ, Walker AJ, West J. Causes of death in people with liver cirrhosis in England compared with the general population: a population-based cohort study. American Journal of Gastroenterology 2015; 110(8):1149-58.

Read 1972

Read AE. Clinical physiology of the liver. British Journal of Anaesthesia 1972;44(9):910-7.

\section{Rice 2017}

Rice JB, White AG, Galebach P, Korenblat KM, Wagh A, Lovelace $\mathrm{B}$, et al. The burden of hepatorenal syndrome among commercially insured and Medicare patients in the United States. Current Medical Research and Opinion 2017; 33(8):1473-80.

\section{Royle 2003}

Royle P, Milne R. Literature searching for randomized controlled trials used in Cochrane reviews: rapid versus exhaustive searches. International Journal of Technology Assessment in Health Care 2003;19(4):591-603.

\section{Salanti 2011}

Salanti G, Ades AE, Ioannidis JP. Graphical methods and numerical summaries for presenting results from multipletreatment meta-analysis: an overview and tutorial. Journal of Clinical Epidemiology 2011;64(2):163-71.

\section{Salanti 2012}

Salanti G. Indirect and mixed-treatment comparison, network, or multiple-treatments meta-analysis: many names, many benefits, many concerns for the next generation evidence synthesis tool. Research Synthesis Methods 2012;3(2):80-97.

Savović 2012a

Savović J, Jones HE, Altman DG, Harris RJ, Jüni P, Pildal $\mathrm{J}$, et al. Influence of reported study design characteristics on intervention effect estimates from randomized controlled trials: combined analysis of meta-epidemiological studies. Health Technology Assessment 2012;16(35):1-82.

\section{Savović 2012b}

Savović J, Jones HE, Altman DG, Harris RJ, Jüni P, Pildal $\mathrm{J}$, et al. Influence of reported study design characteristics on intervention effect estimates from randomized controlled trials. Annals of Internal Medicine 2012;157(6):429-38.

\section{Savovićc 2018}

Savović J, Turner RM, Mawdsley D, Jones HE, Beynon $\mathrm{R}$, Higgins JPT, et al. Association between risk-of-bias assessments and results of randomized trials in Cochrane Reviews: The ROBES Meta-Epidemiologic Study. American Journal of Epidemiology 2018;187(5):1113-22.

\section{Scaglione 2015}

Scaglione S, Kliethermes S, Cao G, Shoham D, Durazo $\mathrm{R}$, Luke A, et al. The epidemiology of cirrhosis in the United States: a population-based study. Journal of Clinical Gastroenterology 2015;49(8):690-6.

\section{Schulz 1995}

Schulz KF, Chalmers I, Hayes RJ, Altman DG. Empirical evidence of bias. Dimensions of methodological quality associated with estimates of treatment effects in controlled trials. JAMA 1995;273(5):408-12.

\section{Setiawan 2016}

Setiawan VW, Stram DO, Porcel J, Lu SC, Le Marchand L, Noureddin M. Prevalence of chronic liver disease and cirrhosis by underlying cause in understudied ethnic groups: the multiethnic cohort. Hepatology (Baltimore, Md.) 2016; 64(6):1969-77.

\section{Severini 1993}

Severini TA. Bayesian interval estimates which are also confidence intervals. Journal of the Royal Statistical Society. Series B (Methodological) 1993;55(2):533-40.

\section{Stata/SE 14.2 [Computer program]} StataCorp LLC. Stata/SE. https://www.stata.com/. Version 14.2. Texas, USA: StataCorp LLC, 2015.

\section{Thorlund 2011}

Thorlund K, Engstrøm J, Wetterslev J, Brok J, Imberger G, Gluud C. User manual for Trial Sequential Analysis (TSA). ctu.dk/tsa/files/tsa_manual.pdf 2011 (accessed 17 July 2018).

\section{Thorlund 2012}

Thorlund K, Mills EJ. Sample size and power considerations in network meta-analysis. Systematic Reviews 2012;1:41.

\section{TSA 2011 [Computer program]} Copenhagen Trial Unit. TSA - Trial Sequential Analysis. Version 0.9.5.10 Beta. Copenhagen: Copenhagen Trial Unit, 2011.

\section{Tsochatzis 2014}

Tsochatzis EA, Bosch J, Burroughs AK. Liver cirrhosis. Lancet 2014;383(9930):1749-61. 


\section{Turner 2012}

Turner RM, Davey J, Clarke MJ, Thompson SG, Higgins JP. Predicting the extent of heterogeneity in meta-analysis, using empirical data from the Cochrane Database of Systematic Reviews. International Journal of Epidemiology 2012;41(3):818-27.

van Valkenhoef 2012

van Valkenhoef G, Lu G, de Brock B, Hillege H, Ades AE, Welton NJ. Automating network meta-analysis. Research Synthesis Methods 2012;3(4):285-99.

\section{Wetterslev 2008}

Wetterslev J, Thorlund K, Brok J, Gluud C. Trial sequential analysis may establish when firm evidence is reached in cumulative meta-analysis. Journal of Clinical Epidemiology 2008;61(1):64-75.

Wetterslev 2017

Wetterslev J, Jakobsen JC, Gluud C. Trial Sequential Analysis in systematic reviews with meta-analysis. $B M C$ Medical Research Methodology 2017;17(1):39.

\section{Williams 2014}

Williams R, Aspinall R, Bellis M, Camps-Walsh G, Cramp M, Dhawan A, et al. Addressing liver disease in the UK: a blueprint for attaining excellence in health care and reducing premature mortality from lifestyle issues of excess consumption of alcohol, obesity, and viral hepatitis. Lancet 2014;384(9958):1953-97.

\section{Wong 2011}

Wong F, Nadim MK, Kellum JA, Salerno F, Bellomo R, Gerbes A, et al. Working party proposal for a revised classification system of renal dysfunction in patients with cirrhosis. Gut 2011;60(5):702-9.

\section{Wood 2008}

Wood L, Egger M, Gluud LL, Schulz KF, Juni P, Altman DG, et al. Empirical evidence of bias in treatment effect estimates in controlled trials with different interventions and outcomes: meta-epidemiological study. BMJ (Clinical Research Ed.) 2008;336(7644):601-5.

* Indicates the major publication for the study

\section{ADDITIONAL TABLES}

\section{Table 1. Criteria for diagnosis of hepatorenal syndrome}

- Diagnosis of cirrhosis and ascites

- Diagnosis of acute kidney injury (AKI) according to International Club of Ascites AKI criteria (ICA-AKI) criteria*

- No response after two consecutive days of diuretic withdrawal and plasma volume expansion with albumin $1 \mathrm{~g}$ per $\mathrm{kg}$ of body weight

- Absence of shock

- No current or recent use of nephrotoxic drugs (non-steroidal anti-inflammatory drugs (NSAIDs), aminoglycosides, iodinated contrast media, etc.)

- No macroscopic signs of structural kidney injury, defined as: absence of proteinuria ( $>500 \mathrm{mg} /$ day), absence of microhaematuria ( $>50$ red blood cells per high-power field), and normal findings on renal ultrasonography. Individuals who fulfil these criteria may still have structural damage such as tubular damage. Urine biomarkers will become an important element in making a more accurate differential diagnosis between hepatorenal syndrome and acute tubular necrosis.

*Increase in serum creatinine $\geq 0.3 \mathrm{mg} / \mathrm{dL}(\geq 26.5 \mu \mathrm{mol} / \mathrm{L})$ within 48 hours or $\geq 50 \%$ increase in serum creatinine from baseline which is known or presumed to have occurred within the previous seven days.

Source: Angeli 2015 
A P PENDICES

\section{Appendix I. Search strategies}

\begin{tabular}{|c|c|c|}
\hline Database & Time span & Search strategy \\
\hline $\begin{array}{l}\text { Central Register of Controlled Trials } \\
\text { (CENTRAL) in the Cochrane Library }\end{array}$ & Latest issue & $\begin{array}{l}\# 1 \mathrm{MeSH} \text { descriptor: [Hepatorenal Syn- } \\
\text { drome] explode all trees } \\
\# 2 \text { hepatorenal syndrom* } \\
\# 3 \# 1 \text { or } \# 2\end{array}$ \\
\hline MEDLINE Ovid & January 1947 to date of search & $\begin{array}{l}\text { 1. exp Hepatorenal Syndrome/ } \\
\text { 2. hepatorenal syndrom*titab. } \\
\text { 3. } 1 \text { or } 2 \\
\text { 4. randomized controlled trial.pt. } \\
\text { 5. controlled clinical trial.pt. } \\
\text { 6. randomized.ab. } \\
\text { 7. placebo.ab. } \\
\text { 8. drug therapy.fs. } \\
\text { 9. randomly.ab. } \\
\text { 10. trial.ab. } \\
\text { 11. groups.ab. } \\
\text { 12. } 4 \text { or } 5 \text { or } 6 \text { or } 7 \text { or } 8 \text { or } 9 \text { or } 10 \text { or } 11 \\
\text { 13. exp animals/ not humans.sh. } \\
\text { 14. } 12 \text { not } 13 \\
\text { 15. } 3 \text { and } 14\end{array}$ \\
\hline Embase Ovid & January 1974 to date of search & $\begin{array}{l}\text { 1. exp hepatorenal syndrome/ } \\
\text { 2. hepatorenal syndrom*.ti,ab. } \\
\text { 3. } 1 \text { or } 2 \\
\text { 4. exp crossover-procedure/ or exp double- } \\
\text { blind procedure/ or exp randomized con- } \\
\text { trolled trial/ or single-blind procedure/ } \\
\text { 5. (((((random* or factorial* or crossover* } \\
\text { or cross over* or cross-over* or placebo* or } \\
\left.\left.\left.\text { double }^{*}\right) \text { adj blind }\right)^{*}\right) \text { or single*) adj blind*) } \\
\text { or assign* or allocat* or volunteer*).af } \\
6.4 \text { or } 5 \\
7.3 \text { and } 6\end{array}$ \\
\hline $\begin{array}{l}\text { Science Citation Index Expanded (Web of } \\
\text { Science) }\end{array}$ & January 1945 to date of search & $\begin{array}{l}\text { \#1 TS= (hepatorenal syndrom*) } \\
\# 2 \text { TS=(random* OR } \text { rct* OR crossover }^{*} \text { OR } \\
\text { OR masked OR blind* OR placebo* OR } \\
\text { meta-analysis OR systematic review* OR } \\
\left.\text { meta-analys }{ }^{*}\right) \\
\# 3 \text { \#1 AND \#2 }\end{array}$ \\
\hline
\end{tabular}

Treatment for hepatorenal syndrome in people with decompensated liver cirrhosis: a network meta-analysis (Protocol) 


\begin{tabular}{|c|c|c|}
\hline $\begin{array}{l}\text { World Health Organization International } \\
\text { Clinical Trials Registry Platform ( } \\
\text { apps.who.int/trialsearch/Default.aspx) }\end{array}$ & $\begin{array}{l}\text { Date of search to be provided at the review } \\
\text { stage }\end{array}$ & Condition: hepatorenal syndrome \\
\hline ClinicalTrials.gov & $\begin{array}{l}\text { Date of search to be provided at the review } \\
\text { stage }\end{array}$ & $\begin{array}{l}\text { Interventional Studies | Hepatorenal Syn- } \\
\text { drome | Phase 2, 3, } 4\end{array}$ \\
\hline $\begin{array}{l}\text { European Medicines Agency } \\
\text { (www.ema.europa.eu/ema/) and US Food } \\
\text { and Drug Administration (www.fda.gov) }\end{array}$ & $\begin{array}{l}\text { Date of search to be provided at the review } \\
\text { stage }\end{array}$ & Hepatorenal syndrome \\
\hline
\end{tabular}

\section{Appendix 2. Sample size calculation}

Approximately $30 \%$ to $60 \%$ of people with hepatorenal syndrome die within a year (Israelsen 2017). The required information size based on a control group proportion of $40 \%$, a relative risk reduction of $20 \%$ in the experimental group, type I error of $5 \%$, and type II error of $20 \%$ is 1128 participants. Network analyses are more prone to risk of random errors than direct comparisons (Del Re 2013). Accordingly, a greater sample size is required in indirect comparisons than in direct comparisons (Thorlund 2012). The power and precision in indirect comparisons depend upon various factors, such as the number of participants included for each comparison and the heterogeneity between the trials (Thorlund 2012). If there is no heterogeneity across the trials, the sample size in indirect comparisons would be equivalent to the sample size in direct comparisons. The effective indirect sample size can be calculated using the number of participants included in each direct comparison (Thorlund 2012). For example, a sample size of 2500 participants in the direct comparison A versus $C\left(n_{A C}\right)$ and a sample size of 7500 participants in the direct comparison $B$ versus $C$ ( $\left.n_{B C}\right)$ results in an effective indirect sample size of 1876 participants. However, in the presence of heterogeneity within the comparisons, the required sample size is higher. In the above scenario, for an $\mathrm{I}^{2}$ statistic for each of the comparisons A versus $\mathrm{C}\left(\mathrm{I}_{A C}{ }^{2}\right)$ and $\mathrm{B}$ versus $\mathrm{C}\left(\mathrm{I}_{B C}{ }^{2}\right)$ of $25 \%$, the effective indirect sample size is 1407 participants. For an $\mathrm{I}^{2}$ statistic for each of the comparisons $\mathrm{A}$ versus $\mathrm{C}$ and $\mathrm{B}$ versus $\mathrm{C}$ of $50 \%$, the effective indirect sample size is 938 participants (Thorlund 2012). If there are only three groups, and the sample size in the trials is more than the required information size, we will calculate the effective indirect sample size using the following generic formula (Thorlund 2012):

$\left(\mathrm{n}_{A C} \times\left(1-\mathrm{I}_{A C}{ }^{2}\right)\right) \times\left(\mathrm{n}_{B C} \times\left(1-\mathrm{I}_{B C}{ }^{2}\right)\right) /\left(\mathrm{n}_{A C} \times\left(1-\mathrm{I}_{A C}{ }^{2}\right)\right)+\left(\mathrm{n}_{B C} \times\left(1-\mathrm{I}_{B C}{ }^{2}\right)\right)$.

Currently, there is no method to calculate the effective indirect sample size for a network analysis involving more than three intervention groups.

\section{CONTRIBUTIONSOFAUTHORS}

Conceiving the protocol: KG, LB.

Designing the protocol: KG, LB.

Co-ordinating the protocol: KG.

Designing search strategies: $\mathrm{KG}$.

Writing the protocol: $\mathrm{LB}, \mathrm{KG}$.

Providing general advice on the protocol: SF, AS, NH, ET.

Securing funding for the protocol: KG.

Performing previous work that was the foundation of the current study: not applicable.

Treatment for hepatorenal syndrome in people with decompensated liver cirrhosis: a network meta-analysis (Protocol) 


\section{DECLARATIONSOF INTEREST}

Lawrence MJ Best: none known.

Suzanne Freeman: none known.

Alex J Sutton: none known.

Neil Hawkins: none known.

Emmanuel Tsochatzis: none known.

Kurinchi Selvan Gurusamy: none known.

\section{SOURCES OF SUPPORT}

\section{Internal sources}

- University College London, UK.

Writing equipment, software, etc.

\section{External sources}

- National Institute for Health Research, UK.

Payment for writing reviews, writing equipment, software 